This is the author's version of an article that has been published in the ICDCM 2019 proceedings.

Changes were made to this version by the publisher prior to publication.

The final version of record is available at https://dx.doi.org/10.1109/ICDCM45535.2019.9232876

\title{
State of Charge Based Characteristic Diagram Control for Energy Storage Systems within Industrial DC Microgrids
}

\author{
Alexander Männel \\ Bosch Rexroth AG \\ Lohr am Main, Germany \\ Email: Alexander.Maennel@boschrexroth.de
}

\author{
Elias Knöchelmann, Svenja Tappe, Tobias Ortmaier \\ Gottfried Wilhelm Leibniz Universität Hannover \\ Institute of Mechatronic Systems \\ Hannover, Germany
}

\begin{abstract}
In this paper, a state of charge (SoC) based characteristic diagram control concept for energy storage systems (ESS) within an industrial direct current (DC) microgrid is proposed. The inputs are the SoC of the ESS and the terminal voltage of the DC microgrid. The output is the charge and discharge current for the converter, which connects the ESS with the DC microgrid. An appropriate design concept for the characteristic diagram is then investigated to achieve a flexible control reacting on changing conditions within the DC microgrid. These could be a temporary overload, due to a changing number of grid participants or an additional feed-in through a photovoltaic (PV) system. The characteristic diagram design concept is even applicable without a deep knowledge of the load profile of the DC microgrid. The concept was analyzed and evaluated based on simulations with load profiles from robot cells. The results show that the SoC depends on the current load of the DC microgrid. If the load returns to average, the SoC of the ESS inclines back to the nominal SoC, which is pre-defined by the grid operator. Furthermore, in case of an appropriate design of the characteristic diagram, it can protect the ESS against overcharging or deep discharging.
\end{abstract}

Index Terms-DC Microgrids, Industrial Production, Energy Storage System, Droop Curve Control, Characteristic Diagram

\section{INTRODUCTION}

A new approach of power supply in production processes are industrial DC microgrids. The main purpose of such grids is to achieve an increased usage of recuperation energy, lower conversion losses and a better response on imbalances of the energy supply and demand compared with state of the art alternative current (AC) technology [1]. One crucial point of DC microgrids is the integration and operation of energy storage systems (ESS), as they balance the load demand and energy supply. However, there are several challenges like the conflict between an optimization of cycle lifetime and a fast amortization of the ESS. On the other hand, flexibility requirements for industrial DC microgrids like connecting or disconnecting grid participants at runtime [1] also challenges the ESS operation. The different number of participants can result in a wide range of different workload situations for the ESS. For this reason, this paper proposes an extension of a common droop curve control for a bi-directional DC-DC converter [2]-[4] for connecting ESSs to the DC microgrid.
The droop curve approach is extended to a three dimensional characteristic diagram with the ESS' $\mathrm{SoC}$ as well as the grids terminal voltage as input values. Other DC microgrid participants can still operate with single input-single output (SISO) droop curve approaches, which for instance use the terminal voltage to compute the converter output current [2].

\section{A. State of the Art}

A typical scheme of an industrial DC microgrid can be seen in Fig. 1. Commonly, the DC microgrid is coupled to the AC grid through an unidirectional or bidirectional ACDC converter. All consumers, which are responsible for the production process, are connected to the DC microgrid as well. Consumers are for instance electrical drives, however they are indicated as four robot arms in Fig. 1. Further DC microgrid participants are energy storage systems (e.g. lithiumion batteries or super capacitors) also connected with gridinterfacing converters. Due to the increasing awareness of energy efficiency, renewable energy sources, such as photovoltaic systems (PV), also became popular for industries. These renewable energy sources can be easily integrated into the DC microgrid. All participants are connected with a DCbus cable, in Fig. 1 indicated as equivalent circuit diagrams. Such an exemplary industrial DC microgrid is also used for the simulation in Section III.

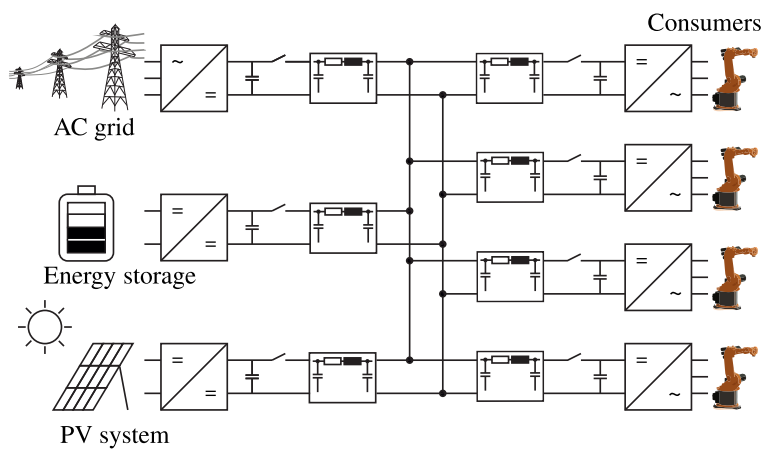

Fig. 1. DC microgrid system.

Copyright (c) 2019 IEEE. Personal use of this material is permitted. For any other purposes, permission must be obtained from the IEEE by emailing pubs-permissions@ieee.org. 
This is the author's version of an article that has been published in the ICDCM 2019 proceedings.

Changes were made to this version by the publisher prior to publication.

The final version of record is available at https://dx.doi.org/10.1109/ICDCM45535.2019.9232876

A common approach for controlling the converters within the DC microgrid is the decentralized droop curve control. These are often applied in DC-powered ships or server data centers [2], [5]. The droop curve can be seen as a virtual impedance of the converters [6]. If transmission line impedances are neglected, the voltages will be equal across the grid. This means that the loads of the consumers can be proportionally shared among the sources through droop curves control [6].

A disadvantage of the droop curve control is the inflexibility regarding changing constraints, such as a volatile power source (PV system) or the SoC of an ESS. In such a case, the droop curve control can lead to an uncontrolled discharging or overcharging of the ESS. To overcome these issues, the adaptive droop curve is proposed in many works [1], [7]. The adaptive droop curve has an additional communication link to a superior control unit. In case of changing constraints, the control unit can adapt the shape of the droop curve to match to the new situation in the DC microgrid. However, the adaptive droop curve requires an additional cabling effort and requires additional hardware and software which increases the complexity. The supposed SoC-based characteristic diagram in this work does not require an additional communication link, as the diagram can be directly evaluated in the converters control. On the other hand, as this approach takes the SoC into account, the diagram control prevents the ESS from overcharging or deep discharging. The flexibility remains, while the cabling effort can be kept low. In [8] the voltage droop curve is extended, resulting in a characteristic diagram which has the electricity costs as a second input. However, a prerequisite is that the electricity costs are accounted variably depending on the current electricity rate. There are already electricity providers which offer variable electricity tariffs for industries [9]. In [4] an adaptive droop curve control is introduced, which switches depending on the SoC of an ESS and results in a droop curve with a hysteresis. Furthermore, it has been shown that all recuperation energy is stored in the ESS and no energy was lost in a breaking resistor. The approach is reasonable in particular if there is no load profile for the consumers given.

In contrary this paper is focused on the introduction of the SoC-based characteristic diagram and a general design concept for those. The remainder of this paper is organized as follows: In Section II the SoC-based characteristic diagram control concept is introduced and a design proposal is shown. After that, the model of an industrial DC microgrid is described in Section III. This model is used in Section IV to analyze and evaluate the design concept in simulation. The proposed approach in this paper is then concluded in Section V.

\section{Concept And Design of SoC-BAsed Characteristic Diagrams}

This work introduces an extension of the voltage based droop curve to a characteristic diagram. The characteristic diagram control concept includes a bi-directional DC-DC converter, which couples the DC microgrid with an ESS, as illustrated in Fig. 2. As one input, the characteristic diagram needs the terminal voltage $u_{\mathrm{DC}}$ of the DC microgrid. The voltage measurement must be filtered. This is necessary to prevent an oversensitive reaction of the characteristic diagram control which can be caused through measurement noise [2]. Like usual SISO droop curve controls, the $u_{\mathrm{DC}}$ can vary in a certain voltage band (e.g. $600 \mathrm{~V}$ to $800 \mathrm{~V}$ ). Appropriate voltage levels are proposed in [1]. The second input is the SoC $s$ of the energy storage. $s$ is estimated and provided by the ESS management system over a communication link to the controller. If the ESS does not provide a SoC estimation, it is also possible to use the storage voltage (e.g. the voltage of a super capacitor). The characteristic diagram is saved as a $3 \mathrm{D}$ look up table in the control unit. Then the controller computes the converter output current $i_{\text {set }}$ with a bilinear interpolation, defined as,

$$
i_{\mathrm{set}}=f\left(\bar{u}_{\mathrm{DC}}, s\right) .
$$

Note that $\bar{u}_{\mathrm{DC}}$ is a low-pass filtered DC microgrid voltage $u_{\text {DC }}$. To achieve more flexibility with respect to changing conditions, it is also possible to re-configure the characteristic diagram by a superior control unit. The droop curve control for other DC microgrid participants can still be used. In order to provide a stable ESS operation with the characteristic diagram, an appropriate dimensioning of the diagram is necessary. Consequently, design strategies of the characteristic diagram are investigated in this chapter. Design objectives that have to be taken into account are the prevention of overcharging or deep discharging of the ESS, while being independent from changing load situations due to varying number of participants within the DC microgrid. The design concept contains two steps. Firstly, a general design of the mains supply droop curve in Section II-A. As the design of the characteristic diagram depends on other supplying participants it is necessary to specify them first. Secondly, the design of the SoC-based characteristic diagram follows in Section II-B.

\section{A. Global properties and mains supply}

For an appropriate design of the SoC-based characteristic diagram, it is necessary to determine global parameters for the gird. Global means that these parameters are valid for the entire DC microgrid. The grid operator must presume those

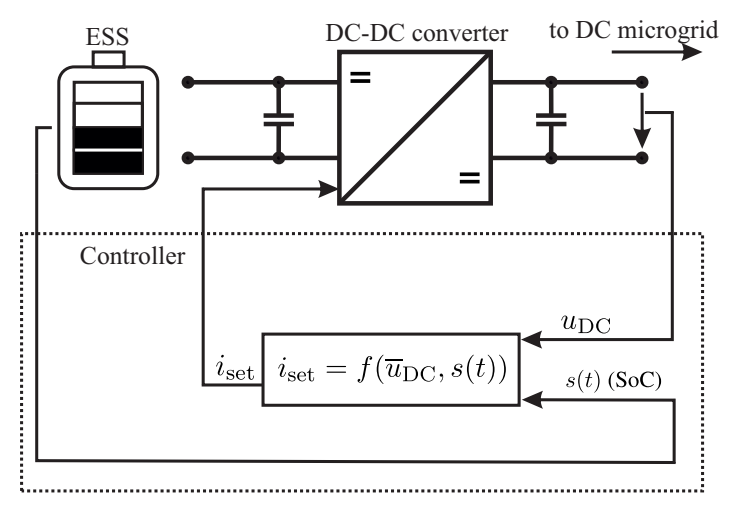

Fig. 2. Control design of the bi-directional DC-DC converter.

Copyright (c) 2019 IEEE. Personal use of this material is permitted. For any other purposes, permission must be obtained from the IEEE by emailing pubs-permissions@ieee.org. 
This is the author's version of an article that has been published in the ICDCM 2019 proceedings.

Changes were made to this version by the publisher prior to publication.

The final version of record is available at https://dx.doi.org/10.1109/ICDCM45535.2019.9232876

parameters in the planning stage. These are sum maximum power $P_{\max }$, the sum minimum power $P_{\min }$, as well as the sum average power $P_{\text {mean }}$ of all consumers connected to the grid. As the $u_{\mathrm{DC}}$ is allowed to vary in a certain range, the maximum and minimum $U_{\max }$ and $U_{\min }$, respectively, and the nominal voltage $U_{\text {nom }}$ have to be defined as global parameters by the grid operator (Table I). $U_{\max }$ and $U_{\min }$ are usually constrained by the devices specification which are part of the DC microgrid. These parameters are used to design the global droop curve. This curve describes the overall system behavior of the DC microgrid [3]. It is the sum of all droop curves in the grid and visualizes the possible power in- and output to cover all the consumer's power demand. An example is shown in Fig. 3. Note that the sign convention is defined as follows: negative current means that the current enters the DC microgrid (supply) and positive currents leave the grid (regenerate). To obtain the global droop curve, the maximum $I_{\max }$, minimum $I_{\min }$ and nominal current $I_{\text {nom }}$ need to be defined:

$$
I_{\min }=-\frac{P_{\max }}{U_{\min }}, I_{\max }=-\frac{P_{\min }}{U_{\max }}, I_{\text {nom }}=-\frac{P_{\text {mean }}}{U_{\text {nom }}} .
$$

$I_{\max }, I_{\min }$ and $I_{\text {nom }}$ are basic points for the global droop curve. Afterwards, the droop curve for the mains supply can be derived. First of all, it has to be defined whether the AC mains interface is unidirectional $(r=0)$ or bidirectional converter $(r=1)$. In case of an unidirectional rectifying, the ESS has to absorb all recuperation energies from the consumers. The grid operator has to decide how much power is contributed by the mains supply with parameter $c_{\text {sup }}(0 \%-100 \%)$. In case of a bidirectional converter, the amount of regeneration absorbed by the mains supply has to be defined as well, with $c_{\text {reg }}(0 \%-100 \%)$. The mean power $P_{\text {mean }}$ is supplied by the mains supply. All parameters for the mains supply droop curve design are listed in Table II. The plant operator is free to choose $c_{\text {sup }}$ and $c_{\text {reg. }}$. The optimal contribution depends

TABLE I

GLOBAL PARAMETERS.

\begin{tabular}{c||c|c} 
Parameter & Variable & Unit \\
\hline Voltage maximum of DC microgrid & $U_{\max }$ & $\mathrm{V}$ \\
\hline Voltage minimum of DC microgrid & $U_{\min }$ & $\mathrm{V}$ \\
\hline Desired nominal voltage of DC microgrid & $U_{\text {nom }}$ & $\mathrm{V}$ \\
\hline Power maximum of all loads & $P_{\max }$ & $\mathrm{kW}$ \\
\hline Power minimum of all loads & $P_{\min }$ & $\mathrm{kW}$ \\
\hline Expected mean power of all loads & $P_{\operatorname{mean}}$ & $\mathrm{kW}$ \\
\hline Capacity of DC microgrid & $C_{\mathrm{DC}}$ & $\mathrm{F}$ \\
\hline
\end{tabular}

TABLE II

MAINS SUPPLY PARAMETERS.

\begin{tabular}{c||c|c} 
Parameter & Variable & Unit \\
\hline Mains supply regeneration & $r$ & true/false \\
\hline Power contribution of regeneration power & $c_{\text {reg }}$ & $\%$ \\
\hline Power contribution of supply power & $c_{\text {sup }}$ & $\%$ \\
\hline
\end{tabular}

on the performance of the ESS. It is planned to optimize those parameters in a future work. After the parameters were determined, the amount of power which is covered by the mains supply can be determined as

$$
I_{\text {min,sup }}=-\frac{c_{\text {sup }} P_{\max }}{U_{\min }}, I_{\max , \text { sup }}=-\frac{r c_{\mathrm{reg}} P_{\min }}{U_{\max }} .
$$

$I_{\text {max,sup }}, I_{\text {min,sup }}$, and $I_{\text {nom }}$ is used as the basic points of the mains supply droop curve. The mains droop curve is illustrated in Fig. 3. One requirement of droop curve and characteristic diagram design is a stable operation under all circumstances. As already mentioned, the droop curve can be seen as the virtual impedance of the converter $Z_{\text {Droop }}$. The reciprocal of those impedance is the droop curve's gain $G_{\text {Droop }}$, as

$$
G_{\text {Droop }}=\frac{1}{Z_{\text {Droop }}} .
$$

In order to guarantee a stable operation, the maximum gain must not exceed a certain level. The maximum gain corresponds to the slope of the droop curve. For this reason, the droop curve designer has to find the highest droop curve slope. In case of the exemplary mains supply droop curve in Fig. 3 (orange line, with regeneration), the highest slope is between $U_{\text {nom }}$ and $U_{\max }$. In addition to the converter's droop curve impedance, the output capacitor's impedance $Z_{\mathrm{C} \text {,out }}$ and the converter's current control loop bandwidth has to be taken into account [2]. With those parameters, a simplified control loop can be set up to perform a stability analysis, as shown in [10]. The analysis can be used to check if $G_{\text {Droop }}$ is in range for a stable grid operation. For details, see [2] and [10].

\section{B. SoC-Based characteristic diagram}

Even though the shape of SoC-based characteristic diagram has to be derived from the global parameters, there are some general properties. First, the characteristic diagram must prevent overcharging or deep discharging of the ESS. As a consequence, for a SoC $s>90 \%$ only discharging is allowed

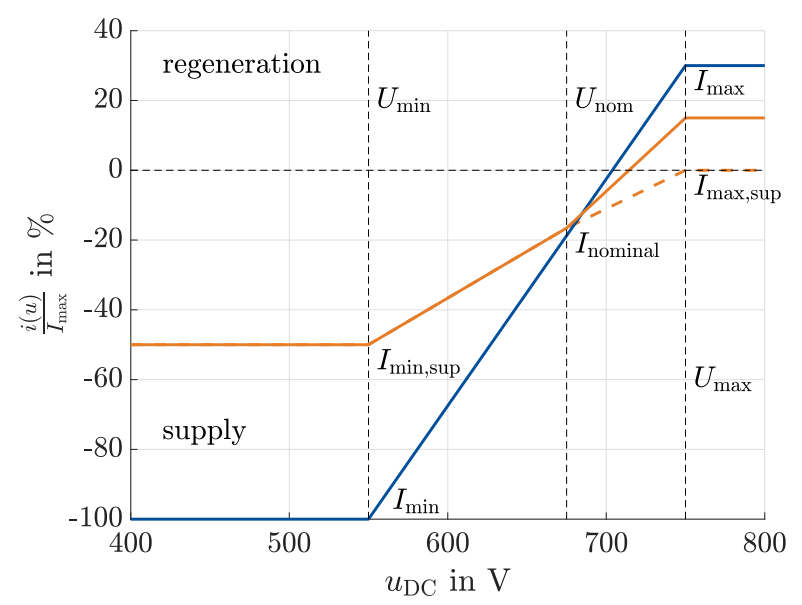

Fig. 3. Global droop curve (blue), droop curve mains supply with regeneration (orange) and droop curve mains supply without regeneration (orange-dashed). 
This is the author's version of an article that has been published in the ICDCM 2019 proceedings.

Changes were made to this version by the publisher prior to publication.

The final version of record is available at https://dx.doi.org/10.1109/ICDCM45535.2019.9232876

and for a SoC $s<10 \%$ only charging. Secondly, as the ESS is supposed to balance the energy supply and demand of the grid, the ESS operates only at peak loads or in case of recuperation. In order to prevent an ESS operation around nominal power load, the characteristic diagram includes a plateau in the center. Exemplary characteristic diagrams are shown in Fig. 4. If the input values $u_{\mathrm{DC}}$ and SoC $s$ are in the area of the plateau, the current output $i_{\text {set }}\left(u_{\mathrm{DC}}, s\right)$ is set to zero. The higher the plateau width $V_{\Delta}$, the bigger is the nocontribution area around the nominal power load. The basic points of the lower plateau boundary $U_{\text {plateau-low }}$ and the higher plateau boundary $U_{\text {plateau-high }}$ can be calculated, as

$$
\begin{aligned}
& U_{\text {plateau-low }}=U_{\text {nom }}-\left(\frac{V_{\Delta}}{2}\right) \text { and } \\
& U_{\text {plateau-high }}=U_{\text {nom }}+\left(\frac{V_{\Delta}}{2}\right),
\end{aligned}
$$

The resulting characteristic diagram can be seen in Fig. 4(a). Furthermore, the plateau can have a shift in the Voltage-SoC plane, as visualized in Fig. 4(b). The plateau shift is beneficial in case of a temporary high or low load situation within the DC microgrid. Due to the plateau shift, the SoC re-adjusts itself back to a defined reference $\mathrm{SoC}\left(S o C_{\text {nom }}\right)$ after the temporary overload situation occurred. In order to calculate these basic points of the plateau shift, an intercept $a$ and and a slope $b$ is necessary. The intercept is obtained, by

$$
a=U_{\text {nom }}-b \cdot S o C_{\text {nom }} .
$$

The parameter $b$ can be chosen by the grid operator. The higher $b$, the faster the self-adjusting of the SoC back to the defined reference $S o C_{\text {nom }}$. In case of $b=0$, the self-adjusting is deactivated. The basic points of the lower plateau boundary $U_{\text {plateau-low }}$, and higher plateau boundary $U_{\text {plateau-high }}$ depending on the SoC $s$ are calculated, as

$$
\begin{aligned}
U_{\triangle, s} & =a+b \cdot s \\
\text { with } \triangle & \in \text { \{plateau-low, plateau-high }\} .
\end{aligned}
$$

The SoC $s$ can range from $0 \%$ to $100 \%$ and $\triangle$ stands for the different basic points of the lower and higher plateau boundary $U_{\text {plateau-low }}$ and $U_{\text {plateau-high }}$. The basic point positions of $U_{\triangle, s}$ are indicated as black lines in Fig. 4(a) and Fig. 4(b). Furthermore, the maximum and minimum current $I_{\mathrm{ESS} \text {,min }}$ and $I_{\mathrm{ESS}, \max }$ contribute the remaining power demand as

$$
I_{\mathrm{ESS}, \min }=-\frac{\left(1-c_{\mathrm{sup}}\right) P_{\mathrm{max}}}{U_{\min }}, I_{\mathrm{ESS}, \max }=-\frac{\left(1-c_{\mathrm{reg}}\right) P_{\min }}{U_{\max }} \text {. }
$$

TABLE III

CHARACTERISTIC DIAGRAM PARAMETERS.

\begin{tabular}{c||c|c} 
Parameter & Variable & Unit \\
\hline Plateau width & $V_{\Delta}$ & $\mathrm{V}$ \\
\hline Nominal SoC at mean load & $S o C_{\text {nom }}$ & $\%$ \\
\hline Plateau shift, intercept & $a$ & - \\
\hline Plateau shift, slope & $b$ & - \\
\hline
\end{tabular}

\section{System Modeling AND Simulation}

An exemplary industrial DC microgrid is used to test the concept. In this section the structure of the grid model is described followed by a mathematical description.

\section{A. Use case}

The modeled DC microgrid is composed of an active front end converter as the bidirectional mains supply. There is a DC-DC converter to control the current from a PV system and an additional converter from a generic ESS with $500 \mathrm{Wh}$ energy and an initial SoC of $50 \%$. The four consumers are load profiles from robot arms, artificially generated by a validated model presented in [11]. The PV profile was taken from [8]. Two different scenarios are performed: Scenario I is without any PV power, however there is a temporary overload between $1000 \mathrm{~s}$ and $4000 \mathrm{~s}$. This overload can be caused due to an additional participant (e.g. an additional robot cell) that is connected at runtime to the DC microgrid. Scenario II includes the standard load profile and an additional PV supply. The profiles of both scenarios can be seen in Fig. 5. The design of the droop curves and the characteristic diagram was obtained

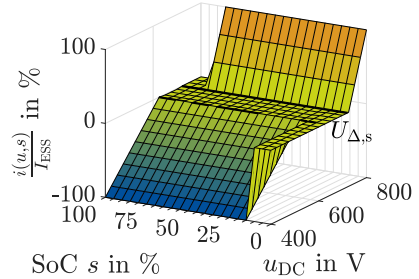

(a) Characteristic diagram $(b=0)$.

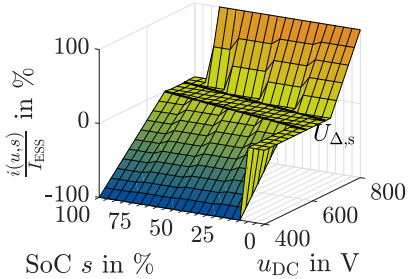

(b) Characteristic diagram $(b=1)$.
Fig. 4. Exemplary characteristic diagrams for energy storage systems. The black lines indicate the basic points $U_{\triangle, s}$ of the diagram plateau.

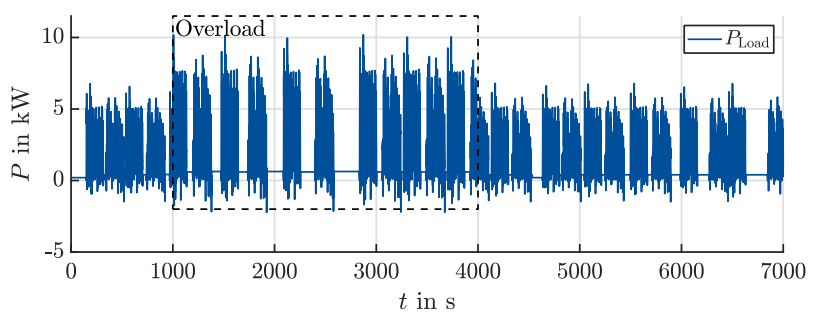

(a) Scenario I with an overload.

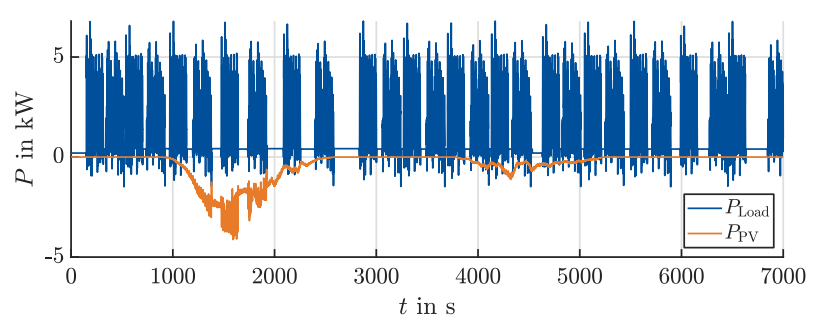

(b) Scenario II with PV power.

Fig. 5. Load and PV profiles of Scenario I and II. 
This is the author's version of an article that has been published in the ICDCM 2019 proceedings.

Changes were made to this version by the publisher prior to publication.

The final version of record is available at https://dx.doi.org/10.1109/ICDCM45535.2019.9232876

according to Section II. The droop curve for the PV system was set to maximum current input. In order to prevent excess voltage in the grid, the droop curve is linearly decreased from $780 \mathrm{~V}$. It was assumed that the load profiles were unknown but only $P_{\max }, P_{\min }$ and $P_{\text {mean }}$ were given. The voltages were set to $U_{\text {nom }}=675 \mathrm{~V}, U_{\min }=400 \mathrm{~V}$ and $U_{\max }=800 \mathrm{~V}$. The supply and regeneration contribution of the AC supply was set to $c_{\text {sup }}=50 \%, c_{\text {reg }}=50 \%$. The characteristic diagram parameters where chosen as follows: Plateau width $V_{\Delta}=60 \mathrm{~V}$, nominal SoC $S o C_{\text {nom }}=50 \%$ and slope $b=2$. The resulting characteristic diagram is visualized in Fig. 6 . The design results for AC supply and PV can be seen in Fig. 7(a) and Fig. 7(b).

\section{B. DC-Grid Modeling}

For simulation, the model introduced in [8] and experimentally validated in [4] was used. Due to cable impedance neglection all capacities in the DC microgrid can be merged into one capacity $C_{\mathrm{DC}}$. The stored energy $E$ in the DC microgrid can be described by the electric field equation for capacitors:

$$
E=\frac{1}{2} \cdot C_{\mathrm{DC}} \cdot U^{2}
$$

Due to the model simplification the applied voltage $U$ of the capacitor is equal to the DC microgrid voltage $u_{\mathrm{DC}}$. In order to calculate the work which is stored in the capacitor at each time

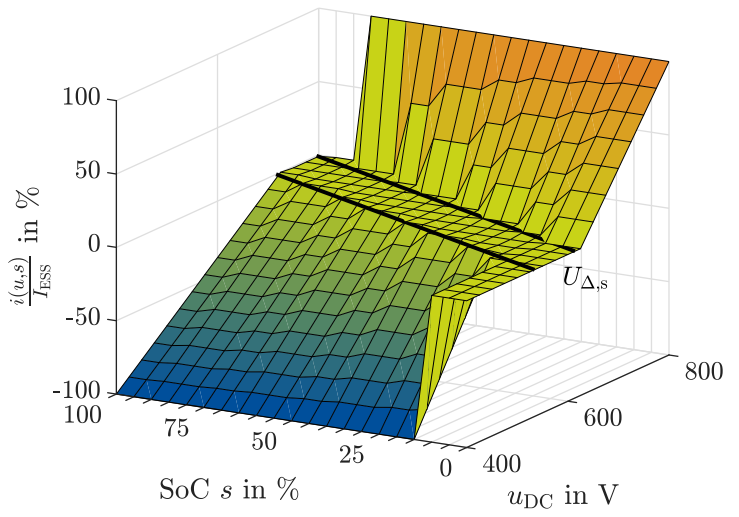

Fig. 6. SoC-based characteristic diagram, normalized. The black lines indicate the basic points $U_{\triangle, s}$ of the diagram plateau.
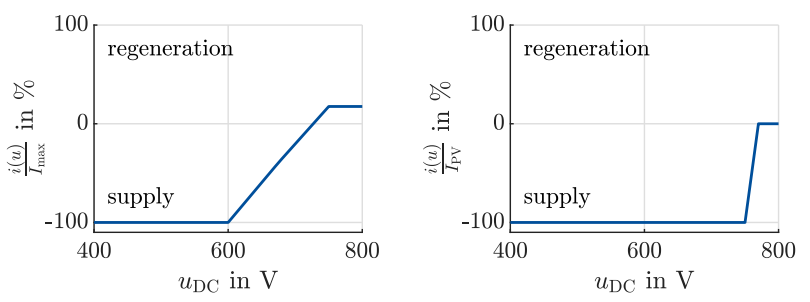

(a) Droop curve of the mains supply. (b) Droop curve of the PV system.

Fig. 7. Droop curves for mains supply and PV. step $k$, the power of all participants has to be summarized to $P_{\text {sum }}$ and then multiplied with the time step $\Delta T$, which yields

$$
\Delta W_{\text {sum }}(k)=P_{\text {sum }}(k) \cdot \Delta T .
$$

For $P_{\text {sum }}$ the current outputs $i_{\text {set }}$ have to be converted to a corresponding power by multiplying it with the DC microgrid voltage. In order to obtain the DC microgrid voltage $u_{\mathrm{DC}}(k)$ Eq. (11) is rewritten, to

$$
u_{\mathrm{DC}}(k)=\sqrt{\frac{2}{C_{\mathrm{DC}}} \cdot\left|\Delta W_{\mathrm{sum}}(k)\right|+u_{\mathrm{DC}}^{2}(k-1)} .
$$

\section{RESULTS}

Both scenarios, introduced in Section II were performed in simulation. The results are shown and evaluated in the following section.

\section{A. Scenario I}

Scenario I has an overload between $t=1000 \mathrm{~s}$ and $t=4000$ s. Fig. 8(a) shows that the DC microgrid voltage $u_{\text {DC }}$ (blue line) drops significantly during this time. As can be seen in Fig. 8(b), the load contribution by the ESS (orange line) increases significantly when temporary overload occurs ( $t=1000 \mathrm{~s}$ to $t=4000 \mathrm{~s}$ ). In contrary, the load contribution of the mains supply slightly increases (blue line). After the overload was over, the ESS demands more power to recharge itself back to nominal SoC $S o C_{\text {nom }}$. This is a result of the plateau shift in the characteristic diagram, which was set to $b=2$. The behavior of the SoC shows Fig. 8(c). As expected, the SoC decreases during the temporary overload. After that,

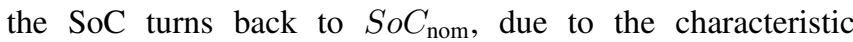
diagram setting.

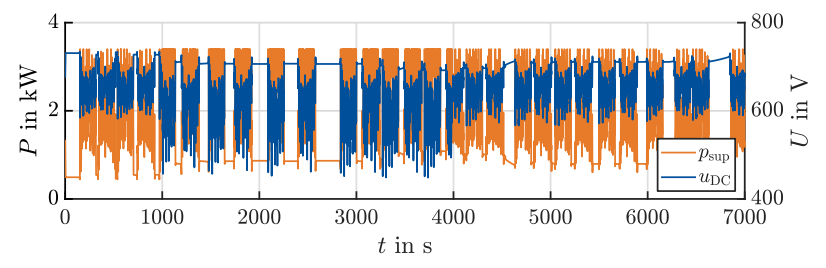

(a) Power of mains supply and DC microgrid $u_{\mathrm{DC}}$.

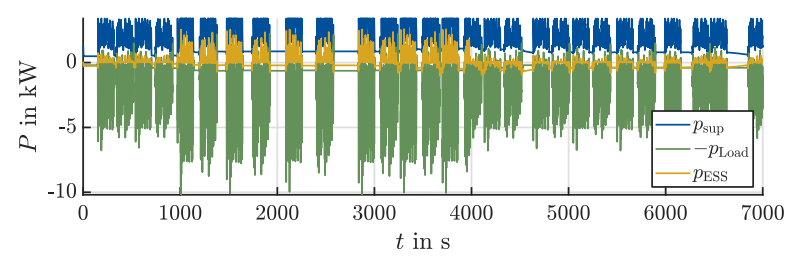

(b) Power of loads, mains supply, PV and ESS.

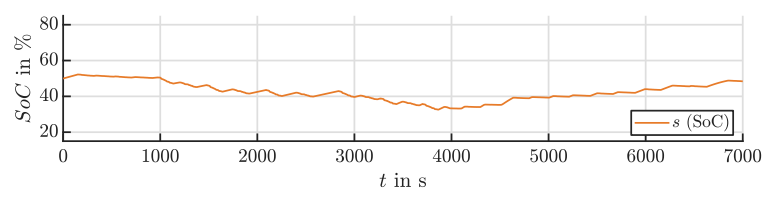

(c) SoC of ESS.

Fig. 8. Simulation results of Scenario I. 
This is the author's version of an article that has been published in the ICDCM 2019 proceedings.

Changes were made to this version by the publisher prior to publication.

The final version of record is available at https://dx.doi.org/10.1109/ICDCM45535.2019.9232876

\section{B. Scenario II}

Scenario II includes a PV supply. There is a large peak around $t=1500 \mathrm{~s}$ and a small peak at $t=4500 \mathrm{~s}$. This leads to a significant increase of the grid voltage $u_{\mathrm{DC}}$, as seen in Fig. 9(a) (blue line). The additional power input from the PV supply is high enough to supply the entire production process. At the first PV power peak, the mains supply contribution turns to zero and erates a small amount of the remaining PV power, back to the AC grid. However, the major amount of the remaining PV power is absorbed by the ESS, as Fig. 9(b) (orange line) shows. After the PV power peak, the ESS has a significantly higher $\mathrm{SoC}$ as $S o C_{\text {nom }}=50 \%$. Due to the characteristic diagram design, the ESS contributes more power to the DC microgrid until the SoC reached $S o C_{\text {nom }}$ again. Like in Scenario I, the characteristic diagram brings the SoC back to $S o C_{\text {nom }}$ after the first PV peak ( $t=1000$ s) as Fig. 9(c) shows. The simulation results show that the characteristic diagram works as expected. Due to the plateau in the diagram, the ESS contributes slightly in normal load conditions, but becomes active in temporary conditions like an overload. The plateau shift with $b=2$ results in a re-adjust of the SoC back to $S o C_{\text {nom }}$ after a temporary condition. The simulation results prove this behavior satisfactorily.

\section{CONCLUSION}

In this paper a SoC-based characteristic diagram control was introduced. This approach is an extension of the voltage based droop curve control for a grid-interfacing bi-directional converter connecting an ESS to a DC microgrid. The inputs of the characteristic diagram are the grid terminal voltage and the

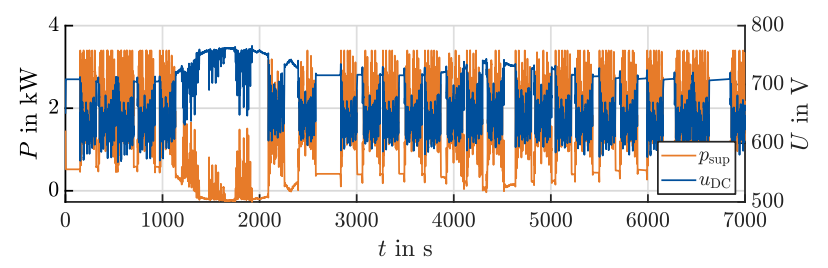

(a) Power of mains supply and DC microgrid $u_{\mathrm{DC}}$.

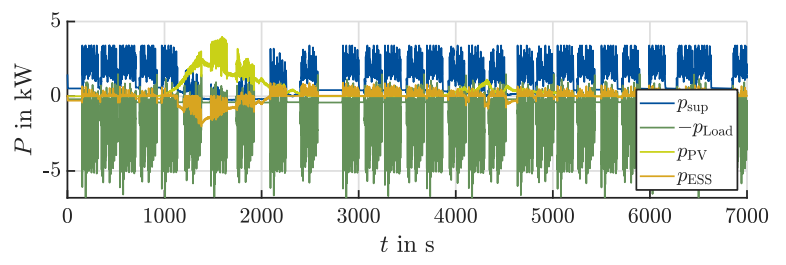

(b) Power of loads, mains supply, PV and ESS.

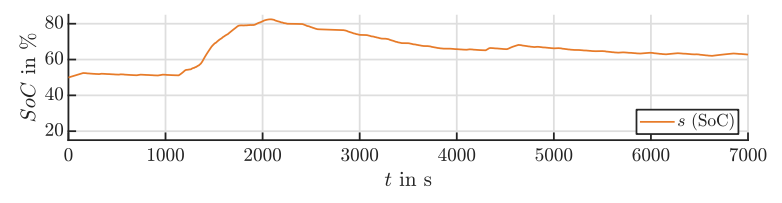

(c) SoC of ESS.
SoC of the ESS to calculate an output current for the converter. It was shown that the characteristic diagram reacts on changing conditions within the grid as requested. The effectiveness has been demonstrated in two scenarios: an overload (e.g. due to a changing number of grid participants) and an additional decentralized feed-in like a PV system. Additionally, the characteristic diagram protects the ESS against overcharging or deep discharging. A future step is a parameter optimization of the characteristic diagram. The goal is to find an optimal plateau width and plateau shift for specific load profiles in order to improve the performance, cycle-lifetime and costs. Even though the simulation has been already validated in [8] and in [4], it is planned to validate the characteristic diagram on a test grid.

\section{ACKNOWLEDGMENT}

This project was part of the DC-INDUSTRIE research project, funded by the German Federal ministry of Economic Affairs and Energy (BMWi).

For further information see: www.dc-industrie.de

\section{REFERENCES}

[1] H. Borcherding, J. Austermann, T. Kuhlmann, B. Weis, and A. Leonide, "Concepts for a dc network in industrial production," in 2017 IEEE Second International Conference on DC Microgrids (ICDCM). IEEE 2017, pp. 227-234.

[2] L. Ott, Y. Han, B. Wunder, J. Kaiser, F. Fersterra, M. Schulz, and M. Marz, "An advanced voltage droop control concept for grid-tied and autonomous dc microgrids," in 2015 IEEE International Telecommunications Energy Conference (INTELEC). IEEE, 2015, pp. 1-6.

[3] D. A. Schaab, S. Weckmann, T. Kuhlmann, and A. Sauer, "Simulative analysis of a flexible, robust and sustainable energy supply through industrial smart-dc-grid with distributed grid management," Procedia CIRP, vol. 69, pp. 366-370, 2018.

[4] A. Männel, S. Tappe, E. Knöchelmann, and T. Ortmaier, "Investigation on an ac grid failure handling of industrial dc micro grids with an energy storage," in 2019 IEEE International Conference on Industrial Technology (ICIT), 2019, pp. 1710-1716.

[5] B. Wunder, L. Ott, J. Kaiser, Y. Han, F. Fersterra, and M. Marz, "Overview of different topologies and control strategies for dc micro grids," in 2015 IEEE First International Conference on DC Microgrids (ICDCM). IEEE, 2015, pp. 349-354.

[6] V. Nasirian, A. Davoudi, and F. L. Lewis, "Distributed adaptive droop control for dc microgrids," in 2014 IEEE Applied Power Electronics Conference and Exposition - APEC 2014. IEEE, 2014, pp. 1147-1152.

[7] T. Dragicevic, X. Lu, J. Vasquez, and J. Guerrero, "Dc microgrids-part i: A review of control strategies and stabilization techniques," IEEE Transactions on Power Electronics, vol. 31, no. 7, pp. 4876-4891, 2016.

[8] E. Knöchelmann, A. Männel, S. Tappe, and T. Ortmaier, "Cost-optimized control of dc microgrids based on characteristic diagrams," in 2019 IEEE International Conference on Industrial Technology (ICIT), 2019, pp. $1685-1691$.

[9] Next Kraftwerke GmbH, "Variable electricity tariffs for industry \& commerce," 2019. [Online]. Available: https://www.nextkraftwerke.de/virtuelleskraftwerk/stromverbraucher/variabler-stromtarif

[10] L. Ott, Y. Han, O. Stephani, J. Kaiser, B. Wunder, M. Marz, and K. Rykov, "Modelling and measuring complex impedances of power electronic converters for stability assessment of low-voltage dc-grids," 2015 IEEE 1st International Conference on Direct Current Microgrids, ICDCM 2015, 2015.

[11] K. Eggers, E. Knöchelmann, S. Tappe, and T. Ortmaier, "Modeling and experimental validation of the influence of robot temperature on its energy consumption," in 2018 IEEE International Conference on Industrial Technology (ICIT), 2018, pp. 239-243.

Fig. 9. Simulation results of Scenario II.

Copyright (c) 2019 IEEE. Personal use of this material is permitted. For any other purposes, permission must be obtained from the IEEE by emailing pubs-permissions@ieee.org. 